\title{
Risco cirúrgico para cirurgias não cardíacas: aspectos práticos
}

\author{
Risk assesment for noncardiac surgery: practical aspects
}

Letícia G. da Rocha

Alfredo de S. Bomfim*

\section{Resumo}

Um frequente motivo de consulta ao cardiologista e ao clínico geral é a avaliação de risco perioperatório em pacientes candidatos a intervenções cirúrgicas não cardíacas. Em última análise, a consulta pré-operatória tem como objetivo identificar e, quando indicado, sugerir intervenções em condições clínicas que possam interferir no resultado da cirurgia. Entretanto, estudos sugerem que essas orientações nem sempre são seguidas pelos consultores, resultando em relatórios incompletos ou meramente burocráticos. A estratificação correta do risco de desenvolver uma complicação cardíaca no período perioperatório permite à equipe médica tomar decisões que contemplam todos os riscos envolvidos, incluído o da doença de base, e buscar as melhores estratégias de tratamento. Nos últimos anos, novas diretrizes têm sugerido uma abordagem escalonada na avaliação do risco cardiológico perioperatório. Tal abordagem leva em consideração o risco inerente ao procedimento proposto, o risco associado à condição cardíaca do paciente e aspectos relativos à doença a ser tratada. Desse modo, busca-se determinar com precisão aqueles pacientes cardiopatas que devem ser tratados antes da cirurgia e quais intervenções realmente interferem no resultado cirúrgico. A presente revisão visa oferecer uma visão sucinta do problema e apontar um modelo prático de avaliação pré-operatória com base nas melhores evidências disponíveis. Algumas situações clínicas específicas comuns em cardiologia também serão comentadas, como forma de auxiliar nas tomadas de decisão.

Descritores: Endocardite; Valva cardíaca; Ecocardiograma; Antibiótico.
Abstract
A frequent reason for consultation to the cardiologist and general practitioner is the assessment of perioperative risk in patients not candidates for cardiac surgery. Ultimately, the preoperative consultation aims to identify and, if indicated, intervene in clinical conditions that 
can affect the outcome of the surgery. However, studies suggest that these guidelines are not always followed by consultants, resulting in underreporting or merely bureaucratic reports. Stratification correct the risk of developing cardiac complications in the perioperative period and allows the medical staff to make decisions that consider all the risks involved, including the underlying disease and to seek the best treatment strategies. In recent years, new guidelines have suggested a stepwise approach in the assessment of perioperative cardiac risk. This approach takes into account the risk inherent in the proposed procedure, the risk associated with the patient's heart condition and aspects of the disease being treated. Thus, we seek to determine precisely those cardiac patients who should be treated before surgery and which interventions truly interfere with the surgical outcome. The present review aims to provide a succinct overview of the problem and point out a practical model for preoperative evaluation based on the best evidence available at present. Some specific common clinical situations in Cardiology will also be commented, as an aid in decision making

Keywords: Perioperative care; Surgical procedures; Operative; Risk.

\section{Introdução}

Nos próximos anos, um aumento no número de procedimentos cirúrgicos não cardíacos é esperado, como decorrência do envelhecimento progressivo da população. ${ }^{1}$ Ao mesmo tempo, sabe-se que a prevalência de doença cardiovascular aumenta com a idade. Esses dados apontam para a necessidade de absorver uma demanda crescente de avaliações pré-operatórias para procedimentos por vezes associados à morbidade e mortalidade significativos. ${ }^{2}$ Estratégias adequadas de avaliação perioperatória têm como objetivo evitar gastos desnecessários de tempo e recursos e, ao mesmo tempo, detectar de forma acurada aqueles pacientes em que há condições que efetivamente possam interferir no manejo clínico do paciente.

Se uma avaliação pré-operatória é solicitada, o papel do médico consultor é identificar as questões fundamentais e certificar-se de que todas as medidas necessárias sejam tomadas por todos os profissionais envolvidos com a realização do procedimento proposto. Entretanto, alguns estudos sugerem que isso nem sempre é feito. Um estudo desenhado para avaliar a percepção de anestesistas, cardiologistas e cirurgiões sobre os propósitos e a utilidade da avaliação pré-operatória mostrou haver grande discrepância entre as diferentes especialidades. ${ }^{3}$ Em uma revisão de 146 consultas para avaliação de risco pré-operatório, apenas em 3,4\% das consultas um novo achado foi identificado. $42 \%$ das consultas não resultaram em nenhuma recomendação. ${ }^{4}$

A carta à equipe cirúrgica deve conter os itens:

- se há doença cardiovascular, sua natureza do estado funcional do coração;

- a probabilidade de ocorrência de complicações e os cuidados necessários para minimizar os riscos;

- a necessidade de interrupção ou modificação dos medicamentos cardioativos em uso;

- a indicação de monitorização intra e pós-operatória;

- a indicação de uso de dispositivos de suporte. A avaliação pré-operatória, como outras formas de consulta médica, começa com história e exame físico cuidadosos. Dados como a presença ou ausência de sintomas específicos, a avaliação da capacidade funcional e o uso de medicamentos são importantes para a tomada de decisão e para a orientação da equipe cirúrgica quanto aos cuidados que devem ser observados. Da mesma forma, comorbidades como doença pulmonar, diabetes, disfunção renal e desordens hematológicas devem ser pesquisadas. Um exame físico cuidadoso deve conter a aferição dos sinais vitais, ausculta cardíaca e pulmonar, exame do abdome e extremidades, palpação dos pulsos e avaliações específicas quando pertinentes. 


\section{Índices multivariados para estimativa de morbidade perioperatória}

A partir dos dados de história e exame físico obtidos, é possível estimar o risco de morbidade perioperatória. Os índices de risco codificam informações clínicas e laboratoriais e alocam pacientes em diferentes categorias de risco. Os índices mais modernos em geral são mais simples e não costumam dar pesos diferentes às diversas condições de risco. $\mathrm{O}$ índice de Lee revisado ${ }^{5}$ é de simples obtenção e tem sido largamente empregado para este fim (tabela 1).

Uma vez obtidas todas as informações necessárias, a tomada de decisão quanto a liberar ou não o paciente para o procedimento proposto pode ser feita através de uma abordagem escalonada por etapas: ${ }^{6}$

primeira etapa - determinar a urgência da cirurgia. Se a intervenção for urgente, não há tempo e nem benefício na realização de exames complementares e o paciente deve ser encaminhado diretamente ao centro cirúrgico;

Tabela 1. Índice revisado de Lee para estimativa de morbidade perioperatória

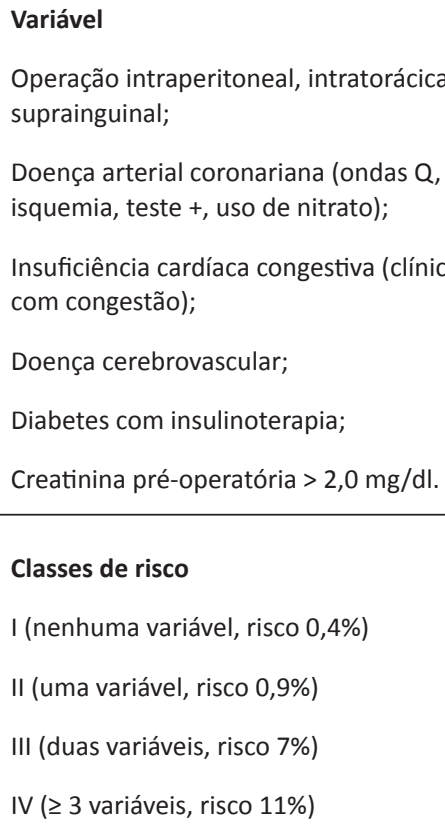

segunda etapa - o paciente possui alguma condição cardíaca ativa? Se sim, essa condição deve ser tratada e estabilizada antes da cirurgia. Estas incluem síndromes coronarianas instáveis, insuficiência cardíaca descompensada, arritmias severas e doença valvar sintomática. Caso não exista nenhuma condição cardíaca ativa, o consultor deve passar à próxima etapa;

terceira etapa - consiste em considerar o risco da cirurgia proposta (tabela 2). Se for baixo, o paciente deve ser encaminhado à cirurgia. Muitos procedimentos estão associados à morbimortalidade de $<1 \%$. $\mathrm{Na}$ realidade, para muitos desses procedimentos, a mortalidade no dia da cirurgia é menor do que no dia 30. Portanto, não há porque adiar. Se o risco do procedimento for moderado ou alto, deve-se proceder à

Tabela 2. Risco associado aos procedimentos cirúrgicos

Alto (risco cardíaco $\geq 5,0 \%$ )

Cirurgias vasculares (aórtica, grandes vasos, vascular periférica);

Cirurgias de urgência ou emergência;

Intermediário (risco cardíaco $\geq 1,0 \%$ e $<5,0 \%$ )

Endarterectomia de carótida e correção endovascular;

Aneurisma de aorta abdominal;

Cirurgia de cabeça e pescoço;

Cirurgias intraperitoneais e intratorácicas;

Cirurgias ortopédicas;

Cirurgias prostáticas

\section{Baixo (risco cardíaco < 1\%)}

Procedimentos endoscópicos;

Procedimentos superficiais;

Cirurgia de catarata;

Cirurgia de mama;

Cirurgia ambulatorial. 
próxima etapa de avaliação;

quarta etapa - determinação da capacidade funcional do paciente. A capacidade funcional se mostrou capaz de prever eventos perioperatórios e de longo prazo. A determinação da capacidade funcional pode ser feita através de um teste ergométrico ou estimada a partir da capacidade de executar tarefas cotidianas (tabela 3). Capacidade funcional igual ou maior que 4 METs é considerada adequada à realização de procedimentos de risco moderado ou mesmo elevado. Em pacientes com capacidade funcional estimada muito baixa (abaixo de 4 METs) a determinação precisa do status funcional é mais difícil através dos questionários de atividade cotidiana e melhor avaliada através de testes ergométricos. Se o paciente for considerado como portador de baixa capacidade funcional, devemos avançar à próxima etapa;

quinta etapa - se o paciente tem capacidade funcional baixa ou indeterminada ou é sintomático, a necessidade de avaliação complementar vai ser determinada pela presença de fatores de risco clínico (tabela 4) e pela natureza da cirurgia. Se houver 1 ou 2 fatores de risco, é razoável realizar a cirurgia, com controle da FC com betabloqueadores. Se houver 3 ou mais fatores de risco, uma intervenção cardíaca pré-operatória está indicada.

\section{Abordagem de condições clínicas específicas}

\section{Doença coronariana conhecida}

Pacientes com doença coronariana conhecida são mais frequentemente testados para pesquisa de isquemia, mas aqueles com doença previamente oculta podem se beneficiar de uma avaliação não invasiva para determinar sua presença. Uma vez determinada a presença de doença coronariana, existem questões importantes a serem respondidas: 1) Quanto de
Tabela 3. Gasto energético estimado para diversas atividades cotidianas

\section{1 a 4 METs}

Vestir-se, comer ou usar o toalete sem ajuda;

Atividades caseiras diárias;

Caminhar ao redor da casa;

Caminhar com 1-2 obstáculos no plano a 3-5 km/h;

\section{5 a 9 METS}

Subir escadas, caminhar em terrenos inclinados;

Caminhar no plano $>6 \mathrm{~km} / \mathrm{h}$;

Correr curtas distâncias;

Atividades moderadas (golfe, dançar, caminhar na montanha).

\section{METS ou mais}

Esportes extremos (natação, tênis, bicicleta).

Trabalho pesado.

Tabela 4: Fatores clínicos de risco perioperatório

História de doença cardíaca isquêmica

História de insuficiência cardíaca;

História de doença cerebrovascular;

Diabetes;

Insuficiência renal.

miocárdio está sob risco? 2) Qual o limiar de isquemia, ou seja, qual a carga de estresse que é necessária para produzir isquemia? 3) Qual a função ventricular? 4) O paciente está em tratamento pleno para isquemia?

Influência da idade e gênero

A idade é um fator relevante, tanto pela maior probabilidade de doença arterial coronariana (DAC) com a idade avançada, quanto pelos efeitos do envelhecimento no miocárdio, que gera aumento da mortalidade por infarto intra e perioperatório neste grupo. ${ }^{7} \mathrm{~A}$ importância do gênero se dá pela menor incidência de DAC no período pré-menopausa, tornando-se equivalente após. ${ }^{8}$ 


\section{Hipertensão arterial sistêmica}

Hipertensão arterial nos estágios 1 e 2 (sistólica $<180 \mathrm{mmHg}$ e diastólica $<110 \mathrm{mmHg}$ ) não são fatores de risco independentes de complicações cardiovasculares no perioperatório. ${ }^{9}$ Não há evidências de que se deva atrasar a cirurgia se não há anormalidades cardiovasculares ou metabólicas. Por isso, a avaliação de risco cirúrgico deve ser considerada uma oportunidade única de identificação desses pacientes para iniciar o tratamento antes do procedimento. A pesquisa de lesão de órgãos-alvo está relacionada à patologia cardiovascular e pode ajudar a definir a cronicidade do quadro.

A isquemia intraoperatória evidenciada no traçado do eletrocardiograma (ECG) se relaciona com maior morbidade cardíaca pós-operatória e pode ser prevenida com a instituição do tratamento para hipertensão no pré-operatório. ${ }^{10}$ Atenção especial deve ser dada a não suspensão do betabloqueador e clonidina por seu efeito rebote, gerando picos hipertensivos.

Para hipertensão no estágio 3 ( $\geq 180$ x 110 $\mathrm{mmHg}$ ), devem ser ponderados potenciais riscos do atraso da cirurgia para controle da pressão arterial, já que se pode lançar mão de agentes intravenosos e conseguir níveis adequados em questão de horas. Se não há comorbidades cardiovasculares significantes, o paciente pode ser submetido ao procedimento, a despeito de pressão arterial elevada no dia da intervenção. ${ }^{11}$

O betabloqueador merece especial atenção, por se mostrar efetivo em modular as variações pressóricas e reduzir a isquemia no pré-operatório, reduzir a incidência de fibrilação atrial no pós-operatório e, para pacientes com risco de DAC, foi eficaz em reduzir mortalidade e a incidência de complicações cardiovasculares durante a hospitalização. ${ }^{12}$

Pacientes em uso de inibidores da enzima conversora de angiotensina (IECAs) e bloqueadores do receptor da angiotensina (BRAs) podem apresentar volume intravascular reduzido e tenderem à hipotensão intraoperatória. Recomenda-se suspender essas medicações na manhã do procedimento e retornar no pós-operatório, assim que se reestabelecer a euvolemia, para evitar risco de disfunção renal. ${ }^{13}$

\section{Insuficiência cardíaca (IC)}

A disfunção ventricular foi apontada em vários trabalhos como relacionada a pior desfecho. É definida como qualquer um dos seguintes: história prévia de insuficiência cardíaca, edema pulmonar, dispneia paroxística noturna, crepitação pulmonar bilateral, presença de B3, RX de tórax com redistribuição vascular pulmonar. A identificação da causa da IC é relevante para definir melhor a estratégia de tratamento e seu prognóstico, por exemplo, IC por DAC tem pior prognóstico que a IC de origem hipertensiva. ${ }^{14}$

\section{Cardiomiopatias}

Deve-se buscar a causa primária da cardiomiopatia, já que as recomendações sobre o manejo pré, intra e pós-operatório serão feitas com base no conhecimento fisiopatológico do processo miocárdico. Isso envolve conhecer a função sistólica e diastólica ventricular através, por exemplo, do ecocardiograma. O principal desafio permanece a cardiomiopatia hipertrófica obstrutiva, pois as variações hemodinâmicas frequentes do intraoperatório incluem redução da volemia e da resistência vascular sistêmica e aumento da capacitância venosa, podendo gerar redução do volume do ventrículo esquerdo e piorando a obstrução da via de saída. ${ }^{15}$ Agonistas beta-adrenérgicos também devem ser evitados por contribuírem com o aumento da obstrução dinâmica e a redução do enchimento diastólico.

\section{Doença valvar}

A avaliação de um sopro no pré-operatório começa em determinar se é um sopro orgânico ou funcional e definir qual paciente fará profilaxia para endocardite e qual deve ser avaliado quanto à gravidade de lesão. Essa avaliação deve ser individualizada e auxiliada por exames complementares, dos quais o ecocardiograma se destaca, por permitir identificar a etiologia da lesão, sua gravidade e por ser um método não 
invasivo e de excelente custo-benefício. Dentre as lesões orovalvares, a estenose aórtica grave é a de mais alto risco para a cirurgia não cardíaca e, sendo o paciente sintomático, esta deve ser adiada ou suspensa para que a lesão valvar seja abordada. ${ }^{16}$

Os pacientes já identificados como portadores de prótese valvar também merecem atenção especial ao tipo de valva, potencial de trombose da mesma, tromboembolismo arterial e risco de endocardite protética. A melhor estratégia de anticoagulação - momento de suspensão do cumarínico, uso de heparina não fracionado ou de baixo peso molecular e sua reintrodução - no pré e pós-operatório deve, de igual forma, ser individualizada de acordo com o risco de sangramento versus risco de tromboembolismo. ${ }^{17}$ Uma discussão mais profunda sobre as lesões orovalvares foge ao tema deste artigo e não será abordada neste momento.

\section{Arritmias e defeitos de condução}

Não são incomuns, especialmente nos idosos. Arritmias supraventriculares e ventriculares foram identificadas como fatores de risco independentes para eventos coronarianos no perioperatório. ${ }^{17}$ Entretanto, achados de arritmia assintomática ao Holter de 24 horas, incluindo taquicardia supraventricular não sustentada (TVNS) não foram associados a aumento de complicações após cirurgias não cardíacas. ${ }^{18}$ Devem, no entanto, levar a uma pesquisa de doença cardiopulmonar de base, isquemia em andamento ou infarto, toxicidade a drogas ou distúrbio metabólico.

Embora relativamente benignas, algumas arritmias supraventriculares podem mascarar problemas cardíacos. Por exemplo, a fibrilação atrial e outras taquiarritmias supraventriculares podem gerar isquemia miocárdica por aumentar a demanda de oxigênio em pacientes com DAC. Por isso, os médicos devem ter um limiar baixo para indicar início de betabloqueador profilático em pacientes com risco aumentado de taquiarritmias no peri ou pós-operatório. Vários estudos sugerem que o betabloqueador pode reduzir a mortalidade e incidência de complicações cardiovasculares, incluindo arritmias durante a cirurgia e até 2 anos depois. ${ }^{19}$

Anormalidades na condução cardíaca, como o bloqueio atrioventricular total (BAVT) podem aumentar o risco operatório e necessitar de marcapasso temporário ou permanente. Por outro lado, pacientes com atraso na condução intraventricular, mesmo na presença de bloqueios de ramo direito ou esquerdo (BRD ou BRE), sem história de bloqueio de avançado ou de sintomas, raramente progridem para BAVT no perioperatório. ${ }^{20} \mathrm{~A}$ disponibilidade de marcapasso transtorácico torna a decisão sobre o transvenoso menos crítica.

Marcapassos (MP) e cardiodesfibriladores implantáveis (CDI)

Devem ser levadas em conta as possíveis interações entre atividade elétrica/magnética gerada pelos MP ou CDI e pela corrente elétrica gerada pelo eletrocautério ou cardioversão, bem como o impacto da disfunção metabólica e agentes antiarrítmicos no MP e seu limiar de sensibilidade.

Embora essa possibilidade de interferência tenha caído drasticamente com o uso de eletrodos bipolares nos dispositivos e com a melhora de seu desenho, ainda podem acontecer, desde reinício do dispositivo temporária ou permanentemente para o modo de back up, até disparos inapropriados do CDI por ativação pelo ruído. O potencial de interferência eletromagnética com o dispositivo é relacionado à quantidade de corrente gerada na vizinhança do MP ou CDI. Em geral, se a via de corrente do cautério está ao longo do eixo do cabo do marcapasso. Antes de procedimento significativo - que inclue cirurgias maiores de abdome ou tórax ou particularmente se envolve uso extenso do eletrocautério - algumas medidas devem ser observadas: determinar qual o tipo de dispositivo implantado, qual sua indicação, se o paciente é dependente dele por terapia antibradicardia e determinar o status da bateria. Aqueles dependentes do marcapasso devem 
ter seu dispositivo avaliado 3 a 6 meses antes e logo após o procedimento, além de ter sua programação alterada para modo assíncrono ou colocar um ímã sobre ele durante a cirurgia. ${ }^{21}$

Medidas referentes ao ambiente operatório incluem: uso do cautério bipolar, se possível; utilização por períodos curtos e intermitentes com o menor nível possível de energia; se unipolar, posicionar a placa terra de forma a minimizar o fluxo de corrente pelo dispositivo; e, se precisar cardioverter, posicionar as pás o mais longe possível e em orientação perpendicular aos cabos do dispositivo - preferir posição anteroposterior.

\section{Recomendações: que teste escolher?}

Para a maioria dos pacientes ambulatoriais, o teste ergométrico é eleito por prover uma estimativa da capacidade funcional e detectar isquemia miocárdica. Aqueles que têm anormalidades importantes no ECG de repouso (BRE, hipertrofia ventricular com padrão strain ou uso de digitálicos) devem ser considerados candidatos a um teste de imagem, como a cintilografia miocárdica com estresse farmacológico ou o ecocardiograma com dobutamina. Dipiridamol e adenosina intravenosos devem ser evitados nos pacientes com relato de broncoespasmo significante ou doença obstrutiva crítica de carótidas. A dobutamina não deve ser utilizada naqueles com história de arritmias graves, hipo ou hipertensão. A indicação de coronariografia é similar àquela fora do contexto de avaliação pré-operatória. As indicações para intervenções coronarianas não devem ser redefinidas simplesmente pela presença de DAC limítrofe e a necessidade do paciente de ser submetido a um procedimento não cardíaco.

\section{Conclusão}

As complicações cardíacas após uma cirurgia não cardíaca são reflexo de fatores específicos do paciente, do procedimento em questão e das circunstâncias sob as quais a cirurgia é realizada. A avaliação pré-operatória pode levar a inter- venções que diminuam o risco perioperatório e a mortalidade em longo prazo ou que alterem o processo de tomada de decisão da cirurgia. Isso pode incluir escolher procedimentos de menor risco, menos invasivos ou optar pelo manejo não operatório. A avaliação de risco pré-operatório deve ser vista pelo clínico ou cardiologista que a realiza, como uma oportunidade de avaliação do paciente que, de outra forma, não realizaria os testes necessários para o seu perfil de risco cardiovascular, independente da necessidade da cirurgia não cardíaca. E, finalmente, um dos objetivos da avaliação pré-operatória é excluir a presença de DAC tão grave que alguma forma de intervenção direta seria justificada, a despeito da necessidade premente da cirurgia não cardíaca.

\section{Referências}

1. Mangano DT. Perioperative cardiac morbidity. Anesthesiology. 1990;72:153-84.

2. Fleisher LA, Eagle KA. Clinical practice: lowering cardiac risk in noncardiac surgery. N Engl J Med. 2001;345:1677-82.

3. Katz RI, Barnhart JM, Ho G, Hersch D, Dayan SS, Keehn L. A survey on the intended purposes and perceived utility of preoperative cardiology consultations. Anesth Analg. 1998;87:830-6.

4. Katz RI, Cimino L, Vitkun SA. Preoperative medical consultations: impact on perioperative management and surgical outcome. Can J Anaesth. 2005;52:697-702.

5. Lee TH, Marcantonio ER, Mangione CM, et al. Derivation and prospective validation of a simple index for prediction of cardiac risk of major noncardiac surgery. Circulation. 1999;100:1043-9.

6. ACC/AHA 2007 Guidelines on Perioperative CardiovascularEvaluation and Care for Noncardiac Surgery. Circulation. 2007;116:e418-e500.

7. GISSI-3: effects of lisinopril and transdermal glyceryl trinitrate singly and together on 6-week mortality and ventricular function after acute myocardial infarction. Gruppo Italiano per lo Studio della Sopravvivenza nell'infarto Miocardico. Lancet. 1994;343:1115-22.

8. Becker RC, Terrin M, Ross R, et al. Comparison of clinical outcomes for women and men after acute myocardial infarction. Ann Intern Med. 1994;120:638-45.

9. Lette J, Waters D, Bernier H, et al. Preoperative and long-term cardiac risk assessment: predictive value of 23 clinical descriptors, 7 multivariate scoring systems, and quantitative dipyridamole imaging in 360 patients. Ann 
Surg. 1992;216:192-204.

10. Chobanian AV, Bakris GL, Black HR, Cushman WC, Green LA, Izzo JL Jr, et al. The Seventh Report of the Joint National Committee on Prevention, Detection, Evaluation, and Treatment of High Blood Pressure: the JNC 7 report. JAMA. 2003;289:2560-72.

11. Weksler N, Klein M, Szendro G, Rozentsveig V, Schily M, Brill S, Tarnopolski A, et al. The dilemma of immediate preoperative hypertension: to treat and operate, or to postpone surgery? J Clin Anesth. 2003;15:17983.

12. Pasternack PF, Grossi EA, Baumann FG, Riles TS, Lamparello PJ, Giangola G, et al. Beta blockade to decrease silent myocardial ischemia during peripheral vascular surgery. Am J Surg. 1989;158:113-6.

13. Colson P, Saussine M, Seguin JR, Cuchet D, Chaptal PA, Roquefeuil B. Hemodynamic effects of anesthesia in patients chronically treated with angiotensin-converting enzyme inhibitors. Anesth Analg. 1992;74:805-8.

14. Cooperman M, Pflug B, Martin EWJ, Evans WE. Cardiovascular risk factors in patients with peripheral vascular disease. Surgery. 1978;84:505-9.

15. Thompson RC, Liberthson RR, Lowenstein E. Perioperative anesthetic risk of noncardiac surgery in hypertrophic obstructive cardiomyopathy. JAMA. 1985;254:2419-21.

16. Otto CM. Valvular aortic stenosis: disease severity and timing of intervention. J Am Coll Cardiol. 2006;47:2141-51.

17. Geerts WH, Pineo GF, Heit JA, Bergqvist D, Lassen MR, Colwell CW, et al. Prevention of venous thromboembolism: the Seventh ACCP Conference on Antithrombotic and Thrombolytic Therapy. Chest. 2004;126:338S-400S.

18. Mahla E, Rotman B, Rehak P, Fruhwald S, Pürstner P, Metzler H, et al. Perioperative ventricular dysrhythmias in patients with structural heart disease undergoing noncardiac surgery. Anesth Analg. 1998;86:16-21.

19. Bayliff CD, Massel DR, Inculet RI, Malthaner RA, Quinton SD, Powell FS, et al. Propranolol for the prevention of postoperative arrhythmias in general thoracic surgery. Ann Thorac Surg. 1999;67:182-6.

20. Pastore JO, Yurchak PM, Janis KM, Murphy JD, Zir LM. The risk of advanced heart block in surgical patients with right bundle branch block and left axis deviation. Circulation. 1978;57:677-80.

21. Practice advisory for the perioperative management of patients with cardiac rhythm management devices: pacemakers and implantable cardioverter-defibrillators: a report by the American Society of Anesthesiologists Task Force on Perioperative Management of Patients with Cardiac Rhythm Management Devices. Anesthesiology. 2005;103:186-98. 